\title{
The pricing kernel puzzle in forward looking data
}

\author{
Horatio Cuesdeanu$^{1}$ (D) J Jens Carsten Jackwerth ${ }^{1}$
}

\begin{abstract}
The pricing kernel puzzle concerns the locally increasing empirical pricing kernel, which is inconsistent with a risk-averse representative investor in a single period, single state variable setting. Some recent papers worry that the puzzle is caused simply by the mismatch of backward looking subjective and forward looking risk-neutral distributions of index returns. By using a novel test and forward looking information only, we generally confirm the existence of a u-shaped pricing kernel puzzle in the S\&P 500 options data. The evidence is weaker for tests against an alternative with a risk-neutral investor and for longer horizons.
\end{abstract}

Keywords Pricing kernel $\cdot$ Option pricing · Forward looking data

JEL Classification G12 - G13

\section{Introduction}

The pricing kernel is a cornerstone of modern finance, as it tells us how to transform subjective probabilities into risk-neutral ones. As the price of any security should be

We received helpful comments and suggestions from Brendan Beare, Peter Christoffersen, Malte Knüppel, Dietmar Leisen, Christian Skov Jensen, Fabian Krüger, Thierry Post, Thorsten Hens, and Axel Kind. We thank seminar participants at the University of Konstanz, the University of Lancaster, the University of Toronto, the University of Sydney, and the University of Queensland.

$\bowtie$ Horatio Cuesdeanu

horatio.cuesdeanu@uni-konstanz.de

Jens Carsten Jackwerth

jens.jackwerth@uni-konstanz.de

1 University of Konstanz, PO Box 134, 78457 Konstanz, Germany 
the discounted expected payoff under the risk-neutral measure, the pricing kernel is indispensable for pricing. By assuming a simple single-period, representative investor economy, one can show that the pricing kernel is proportional to marginal utility. ${ }^{1}$ Assuming risk-aversion in such a setting results in a monotonically decreasing pricing kernel. ${ }^{2}$ Empirically, researchers use a large index, e.g. the S\&P 500, as a reasonable proxy for end-of-period wealth. Yet, when empirically estimating the pricing kernel, it turns out to be locally increasing, which is inconsistent with the above economy as that would imply risk-seeking behavior. Ait-Sahalia and Lo (2000), Jackwerth (2000), and Rosenberg and Engle (2002) were the first to find this discrepancy and labeled it the pricing kernel puzzle.

We do not literally suggest the existence of risk-seeking behavior, but the empirical evidence shows a need for more encompassing models capable of generating the pricing kernel puzzle while maintaining the assumption of a risk-averse investor. Chabi-Yo et al. (2009), Bakshi et al. (2010) and Benzoni et al. (2011) describe such models. ${ }^{3}$ Through the lens of these richer models, the true (multi-dimensional) pricing kernel, when projected onto market returns, turns out to be locally increasing. We nevertheless stick to the wording "pricing kernel puzzle" as it became standard in the finance literature to describe empirical pricing kernels, which locally increase in the market return.

The standard approach for obtaining empirical pricing kernels is as follows. The risk-neutral density is backed out from the forward looking option prices written on the index. The subjective density is inferred from past index returns using non-parametric or parametric models. Finally, the (discounted) ratio of the risk-neutral divided by the subjective density delivers the pricing kernel. Jackwerth (2004) and Cuesdeanu and Jackwerth (2017) survey the literature and detail the different approaches, which tend to confirm the pricing kernel puzzle using slightly different methods and data sets. More recently, Beare and Schmidt (2014b), using a novel statical test, and Babaoglu et al. (2017) confirm the pricing kernel puzzle yet again in the data.

Only a few studies find evidence for monotonically decreasing pricing kernels, and we group them into three categories. Each of the groups throws up problems, which we want to address in our work. First, Bliss and Panigirtzoglou (2004) start out with pricing kernels based on power or exponential utility functions and thus restrict pricing kernels to monotonically decrease by construction. Instead, we allow both unrestricted pricing kernels and ones, which are restricted to monotonically decrease. Second, Barone-Adesi et al. (2008) and Barone-Adesi et al. (2012) argue that their pricing kernels are broadly decreasing even though they are locally increasing. This begs for a formal statistical test of monotonicity, which can distinguish between insignificant local fluctuation and significant non-monotonicity of the pricing kernel. Beare and

\footnotetext{
1 See e.g. (Cochrane 2000, p. 50).

2 Dybvig (1988) and Beare (2011) provide another interesting view on the puzzle under the assumption of a complete market. Buying the market portfolio is the cheapest way to obtain the payoff of the market portfolio if and only if the pricing kernel is monotonically decreasing. Or stated differently, if the pricing kernel is locally increasing, there exists a portfolio of Arrow-Debreu securities that stochastically dominates the market return. See also Beare and Schmidt (2014a) for an empirical study on this issue.

3 For additional models of the pricing kernel puzzle, see Cuesdeanu and Jackwerth (2017).
} 
Schmidt (2014b) propose such tests when the subjective density is inferred from past returns. We provide monotonicity tests when, instead, using forward looking data only.

Third, Linn et al. (2017) voice concern about the usual methodology, which mixes forward looking information for the risk-neutral density and backward looking information for the subjective density. As usual, they find the risk-neutral density from option prices based on a standard forward looking method. To avoid using backward looking subjective densities, they assume a (potentially increasing) pricing kernel based on B-splines, which allows them to compute the forward looking subjective density. ${ }^{4}$ They leave the resulting subjective density unscaled, so that the probabilities do not need to add up to one. They then work out the likelihood that a realized return over the next month is drawn from the subjective distribution. Maximizing the aggregated likelihood over many months allows them to find the pricing kernel that is most consistent with the realized future returns. According to the authors, the best pricing kernels are monotonically decreasing and hence consistent with economic theory. It is somewhat surprising that their pricing kernels do not increase even locally due to some noise in the data. Note that the authors do not test for monotonicity. We argue in Sect. 5.5 that their surprising results could be driven by the lack of scaling and their particular combination of different objective functions for the data fit.

Given the contradicting evidence, is the pricing kernel puzzle really a feature of the data? To answer this question, we suggest a number of methodological improvements and a novel test for pricing kernel monotonicity when using forward looking information only. For one, we use forward looking data only as we share the concern of Bliss and Panigirtzoglou (2004) and Linn et al. (2017) that historical index return data might not be informative about the forward looking subjective distribution in the presence of regime shifts or non-stationary distributions. Second, we use flexible pricing kernels, which allow for increasing segments, in line with Linn et al. (2017) and extending the methodology of Bliss and Panigirtzoglou (2004). Picking a tentative pricing kernel, we can obtain the forward looking subjective density by dividing the forward looking risk-neutral density by the assumed pricing kernel. We can now find the log score of the observed future return on the market. ${ }^{5}$ Maximizing the average log scores gives us the best unrestricted pricing kernel. Maximizing the average log scores, while requiring the pricing kernel to monotonically decrease, gives us the best restricted pricing kernel. Third, we provide a formal test of pricing kernel monotonicity by simulating the difference in average log scores between restricted and unrestricted pricing kernels. We extend our test to a number of alternative parametric and non-parametric test statistics measuring the quality of the pricing kernel to explain the data. Namely, we use, as alternatives to the log score and in line with Bliss and Panigirtzoglou (2004) and

\footnotetext{
4 B-splines are scaled truncated power functions, which can be used instead of polynomial splines (de Boor 1978, p. 96). Their theoretical characterization makes them favorable for the estimation procedure in Linn et al. (2017).

5 The methodology applied in Bliss and Panigirtzoglou (2004), Linn et al. (2017), as well as in our paper is forward looking in the sense that no historical return time series is used to explicitly compute the subjective density. Nevertheless, monthly realized returns are needed for calibrating the pricing kernel, see Sect. 2.3. Recovering the pricing kernel without using past returns but relying on more strict assumptions on the data generating process was pioneered by Ross (2015); see also the follow-up studies by Jackwerth and Menner (2017) and Jensen et al. (2017).
} 
Linn et al. (2017), the moment fit criterion presented in Knüppel (2015), the Cramér van Mises statistic, and the Berkowitz (2001) test.

Existing pricing kernel monotonicity tests confirmed the existence of the pricing kernel puzzle but used different assumptions and data sets. Examining options written on the German market index on three dates at the end of June 2000, 2002, and 2004, Golubev et al. (2014) reject pricing kernel monotonicity. Their approach is similar to ours, in that they avoid the explicit estimation of subjective densities inferred from historical return data. However, this comes at the cost of assuming iid returns and, hence, unconditional subjective and risk-neutral distributions at each of the three dates, which have been picked in order to represent different market regimes: a bull market, a stable market, and a bear market. Bakshi et al. (2010) and Chaudhuri and Schroder (2015) avoid the estimation of subjective densities as well by deriving testable implications for option returns under the assumption of a monotonically decreasing pricing kernel. Both articles find monotonicity violations when applying their tests to index option data. Beare and Schmidt (2014b) allow for conditional distributions and still find monotonicity violations in the S\&P 500 data with two tests based on ordinal dominance curves. Unfortunately, their use of historical returns for obtaining the subjective distribution exposes their results to the criticism of mixing forward and backward looking information.

Testing pricing kernel monotonicity with only forward looking information has an important economic implication. If the pricing kernel in such setting would turn out to be monotonically decreasing, the puzzle could be easily explained by the fact that researchers used biased, backward looking estimates of the subjective distribution (e.g., GARCH, stochastic volatility models, kernel density). In addition, all existing monotonicity tests mixing forward and backward looking information would have to be seen very critically as well.

Yet, using our new test and only forward looking data, we confirm the pricing kernel puzzle in the data. Our findings are stable for different samples and goodness of fit criteria, including the ones used in Bliss and Panigirtzoglou (2004) and Linn et al. (2017). Consistent with the recent theoretical and empirical literature, the estimated pricing kernel displays an overall u-shape (Bakshi et al. 2010; Christoffersen et al. 2013) with further non-monotonicities in the center (Cuesdeanu 2016). In our most conservative test, which tests against an alternative assuming a risk-neutral investor, two out of four goodness of fit criteria still reject pricing kernel monotonicity at the monthly horizon. Considering longer horizons, 2 and 3 months, it is interesting to note that pricing kernel monotonicity cannot be rejected.

The remainder of the paper proceeds as follows. Section 2 outlines our empirical approach and the construction of potential pricing kernels. Section 3 describes the data set. In Sect. 4, we present the results when the pricing kernel is modeled as a piece-wise linear function. Results from various robustness checks are provided in Sects. 5 and 6 concludes. 


\section{Methodology}

We identified the desirability of a forward looking assessment of the subjective probability distribution, which is typically unknown. What we have readily available instead is the option implied risk-neutral density, which, in a single period representative agent economy as in e.g. Cochrane (2000, p. 50), is linked to the subjective density via the pricing kernel. Formally, the subjective density is given by:

$$
p_{t}\left(R_{t}\right)=\frac{q_{t}\left(R_{t}\right)}{m\left(R_{t}\right)}
$$

where $p_{t}\left(R_{t}\right)$ denotes the time $t$ conditional subjective density for the rate of return realized over the following month $R_{t}=S_{t+\Delta t} / S_{t}$ with $S_{t}$ being the stock price at time $t$. The time $t$ conditional risk-neutral density is denoted by $q_{t}\left(R_{t}\right)$ and the pricing kernel is $m\left(R_{t}\right)$. We assume that the pricing kernel, and hence preferences, are constant through time. This assumption does not imply stationarity for the subjective or riskneutral distribution. Both distributions are allowed to vary over time; only the ratio of the densities has to be the same. The constant preferences assumption is essential for the approach used in this study and was also applied by Bliss and Panigirtzoglou (2004) and Linn et al. (2017). Being worried that the pricing kernel might change somewhat over time, we show in Sect. 5 that our results survive for different subsamples and the shape of the pricing kernel does not change extensively.

We next discuss the estimation and construction of the two ingredients of the subjective density as given in Eq. (1): the risk-neutral density $q_{t}$ and the pricing kernel $m$.

\subsection{The risk-neutral density}

Empirically, we need to estimate the risk-neutral density $\widehat{q}_{t}$ of monthly returns, which we obtain from the fast and stable method of Jackwerth (2004), which uses average implied volatilities based on bid and ask option prices. The method then finds implied volatilities $\left\{\sigma_{j}\right\}_{j=0}^{J}$ on a fine grid of 2500 moneyness levels from 0.2 to 1.8 and trades off between fitting a smooth implied volatility function while matching the implied volatilities observed in the market. The optimal implied volatilities are given by the closed form solution (given the trade-off parameter $\lambda$ ) of the following minimization problem:

$$
\min _{\sigma_{j}} \frac{\delta^{4}}{2(J+1)} \sum_{j=0}^{J}\left(\sigma_{j}^{\prime \prime}\right)^{2}+\frac{\lambda}{2 I} \sum_{i=1}^{I}\left(\sigma_{i}-\bar{\sigma}_{i}\right)^{2}
$$

Thus, we obtain $J+1$ implied volatilities $\sigma_{j}$ corresponding to a grid consisting of $J+1$ strike prices $K_{j}$. The fineness of the grid is given by $\delta$, which is the difference between two successive strikes in the grid. The index $i$ in the second term of Eq. (2) represents those implied volatilities $\sigma_{i}$, at which an option with implied volatility $\bar{\sigma}_{i}$ for the strike price $K_{i}$ is observed in the market. The trade-off between smoothness and fit is controlled by the parameter $\lambda$. The smooth implied volatility curve is translated into the estimated risk-neutral density $\widehat{q}_{t}$ by means of the Breeden and Litzenberger 
(1978) result. Our results are robust to using instead the fully parametric method of Gatheral (2004) for the risk-neutral density, see Sect. 5.3.

\subsection{Construction of potential pricing kernels}

We model a tentative pricing kernel $\tilde{m}$ as a piece-wise linear function and, in a robustness test, as a cubic spline to obtain a smooth pricing kernel. Setting up monotonicity constraints is straightforward in the linear case and so we start with this setting. For specifying a tentative pricing kernel, we fix nine equally spaced values on the return axis: $0.8,0.85,0.9,0.95,1,1.05,1.1,1.15$, and $1.2 .{ }^{6}$ We fix the pricing kernel at a return of 0.8 at a value of 5 , allow each of the other eight points to take pricing kernel values between zero and five, and interpolate linearly. Changing the fixed value at 0.8 away from 5 and changing the upper limit has virtually no effect on our results since the subjective densities are rescaled, see Sect. $2.3 .^{7}$ As we only use option quotes with moneyness $\left(=K / S_{t}\right.$ ) between 0.8 and 1.2, we follow Rosenberg and Engle (2002) and set the pricing kernel below 0.8 to its value at 0.8 and above 1.2 to its value at 1.2. ${ }^{8}$ Hence, in our base setting, a tentative pricing kernel $\widetilde{m}\left(R_{t} ; \Theta\right)$ is a piece-wise linear function of eight parameters $\Theta=\left\{\theta_{1}, \ldots, \theta_{8}\right\}$, which correspond to the values of the pricing kernel at the returns $0.85,0.9,0.95,1,1.05,1.1,1.15$, and 1.2.

\subsection{The subjective density}

Varying the parameters $\Theta$ leads to a tentative pricing kernel $\tilde{m}$, which we can combine with our estimate of the risk-neutral density $\widehat{q}_{t}$, so that we obtain the subjective density as

$$
\tilde{p}_{t}\left(R_{t} ; \Theta\right)=\frac{\widehat{q}_{t}\left(R_{t}\right)}{\widetilde{m}\left(R_{t} ; \Theta\right)} / \int_{0}^{\infty} \frac{\widehat{q}_{t}\left(R_{t}\right)}{\widetilde{m}\left(R_{t} ; \Theta\right)} d R_{t} .
$$

The integral in Eq. (3) scales the subjective density such that it integrates to unity as probabilities should sum up to one. ${ }^{9}$ Bliss and Panigirtzoglou (2004) perform the same normalization while Linn et al. (2017) do not scale the subjective density, leading them to a monotonically decreasing pricing kernel estimate, see Sect. 5.5.

Given some tentative pricing kernel $\tilde{m}\left(R_{t} ; \Theta\right)$, we can now assess the log score of the future realized return in terms of a tentative subjective density $\ln \left(\widetilde{p}_{t}\left(R_{t} ; \Theta\right)\right) .{ }^{10}$

\footnotetext{
6 Using a different number of equally spaced values on the return axis does not change the results, see Sect. 5.2 .

7 Consider also Figs. 1, 2, and 3 where such estimated pricing kernels are depicted. After scaling the pricing kernel, it can take on any value and is therefore not restricted by the initial value of 5 .

8 Our method is robust to extending the pricing kernel below 0.8 and above 1.2 at the slopes of the last interior segments instead of horizontally. Moreover, the results do not change when fixing different basis points on the return axis, see Sect. 5.2

${ }^{9}$ Our formulation is equivalent to expressing the subjective density in terms of state prices $\pi_{t}$ as the riskfree discount factor $e^{-r_{f, t} \Delta t}$ cancels out when normalizing the subjective densities:

$p_{t}=\frac{\pi_{t}}{m} / \int_{0}^{\infty} \frac{\pi_{t}}{m} d R_{t}=\frac{e^{-r} f, t}{m} q_{t} / \int_{0}^{\infty} \frac{e^{-r_{f, t} \Delta t} q_{t}}{m} d R_{t}=\frac{q_{t}}{m} / \int_{0}^{\infty} \frac{q_{t}}{m} d R_{t}$.

10 See Gneiting and Raftery (2007) for a comprehensive discussion of score functions.
} 


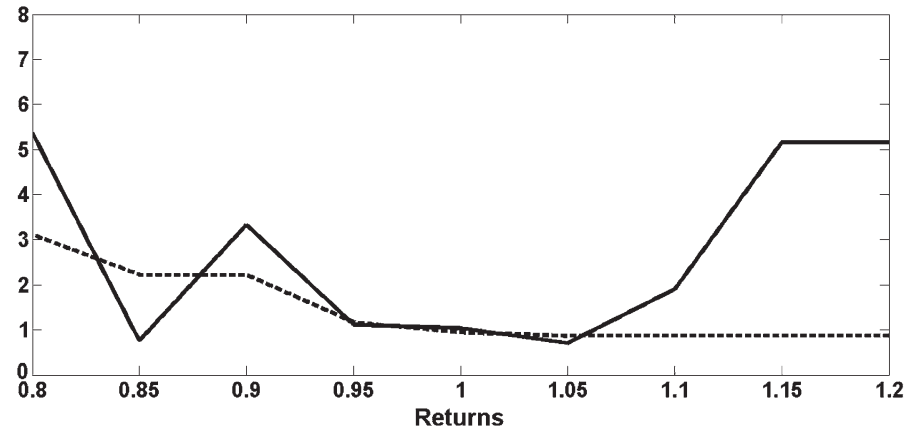

Fig. 1 Linearly interpolated pricing kernels. We plot an unrestricted scaled pricing kernel (solid) and a non-increasing scaled pricing kernel (dotted), constructed by our linear interpolation rule and maximizing the average log score as given in Eq. (4). Both pricing kernels are scaled by the risk-neutral density from 28th January 2015. We observe that the best fitting unrestricted pricing kernel has increasing regions. Returns are on the horizontal axis, and the values of the pricing kernel are on the vertical axis

The sample mean of the log scores gives us the average log score and measures how likely the observed returns were drawn from the tentative density, with a high value indicating a high likelihood. ${ }^{11}$

$$
\widetilde{L S}(\Theta)=\frac{1}{N} \sum_{t=1}^{N} \ln \left(\widetilde{p}_{t}\left(R_{t} ; \Theta\right)\right)=\frac{1}{N} \sum_{t=1}^{N} \ln \left(\frac{\widehat{q}_{t}\left(R_{t}\right)}{\widetilde{m}\left(R_{t} ; \Theta\right)}\right)
$$

where $N$ is the number of (monthly) observations.

By varying the tentative pricing kernel over its parameters $\Theta$, we maximize the average log score $\widetilde{L S}(\Theta)$ subject to the no-arbitrage condition $\left(\widetilde{m}>0\right.$, i.e., $\theta_{i}>$ 0 for $i=1, \ldots, 8)$ and call the result the unrestricted pricing kernel $\widehat{m}^{\text {unrestr. }}$.

In a second maximization, we add the condition that the pricing kernel ought to be monotonically decreasing ( $\tilde{m}^{\prime} \geq 0$, i.e., $\theta_{i} \geq \theta_{j}$ for $\left.i \leq j\right)$, so the result is consistent with an increasing and concave utility function. We call this result the restricted pricing kernel $\widehat{m}^{\text {restr. }}$.

Figure 1 anticipates our main result and illustrates such tentative pricing kernels constructed by our linear interpolation rule. In particular, the unrestricted (restricted) pricing kernel in this figure is the best fitting unrestricted (restricted) pricing kernel when maximizing the average log score, as given in Eq. (4), see Sect. 4 for details. Note that the best fitting unrestricted pricing kernel has pronounced increasing regions and, hence, is not monotonically decreasing. The average log score is but one measure, which can be used to estimate unrestricted and restricted pricing kernels by optimizing the fit of the corresponding subjective densities to the data, and we detail alternatives in Sect. 2.5. We now turn to describing our formal test of monotonicity of the pricing kernel.

\footnotetext{
11 A similar approach is used in Shackleton et al. (2010) for mixing forecasts from past returns and forward looking option prices.
} 


\subsection{Monotonicity test}

We find that our average log score $(L S)$ is 2.00 for the best-fitting (unrestricted) pricing kernel and 1.98 for the non-increasing (restricted) pricing kernel. We are thus left with the question, if the difference $\Delta_{L S}:=L S^{\text {unrestr. }}-L S^{\text {restr. }}$ is statistically significant? As this difference is zero if and only if the true $m$ is decreasing, we formulate our null hypothesis as:

$$
H_{0}: m \text { is non-increasing }
$$

As the distribution of the difference $\Delta_{L S}$ is unknown, we address this problem by simulating the distribution similarly to Bliss and Panigirtzoglou (2004). To be conservative, we follow the latter and Beare and Schmidt (2014b) by simulating the $\Delta_{L S}$ distribution by assuming a constant (i.e., risk-neutral) pricing kernel. Additionally, we explore situations where the true pricing kernel $m^{\text {power }}\left(R_{t}\right)=R_{t}^{-\gamma}$ stems from a power utility function with a fixed $\gamma \geq 0$ and, hence, the true pricing kernel is non-increasing. For each month, we obtain the subjective density $p_{t}^{\text {power }}$ consistent with our power utility function by dividing the observed risk-neutral density for that month by the power utility pricing kernel, as defined in Eq. (3)

$$
p_{t}^{\text {power }}\left(R_{t}\right)=\frac{\widehat{q}_{t}\left(R_{t}\right)}{m^{\text {power }}\left(R_{t}\right)} / \int_{0}^{\infty} \frac{\widehat{q}_{t}\left(R_{t}\right)}{m^{\text {power }}\left(R_{t}\right)} d R_{t}
$$

Afterwards, we draw returns from these subjective densities, one from each monthly distribution. Finally, we employ our usual methodology and search for the best unrestricted and restricted pricing kernels which minimize the average log score. We calculate the differences $\Delta_{L S}$ and repeat the procedure 10,000 times; generating the distribution of $\Delta_{L S}$ under the null. We use this distribution to find the $p$ value of the empirical $\widehat{\Delta}_{L S}$, which we obtain from the data.

We employ the risk-aversion coefficients $\gamma=0, \gamma=2$, and $\gamma=4$. The latter value models a risk-averse investor and is close to the estimate of $\gamma=4.08$ in Bliss and Panigirtzoglou (2004), based on a monthly horizon. From a statistical point of view, Beare and Schmidt (2014b) argue that monotonicity tests should use risk-neutrality (i.e., $\gamma=0$ and $m^{\text {power }}=1$ ) because this is the point in the null at which it is most likely to reject the null. Hence, setting critical values at $m^{\text {power }}=1$ allows to control the Type I error for all other points in the null. ${ }^{12}$ The risk-neutral density is then equal to the subjective density and a rejection of non-monotonicity is very difficult since a flat pricing kernel is the least decreasing pricing kernel specification and therefore most distant from the null hypothesis. ${ }^{13}$

\footnotetext{
12 We thank an anonymous referee for stressing this point.

13 See Beare and Moon (2015) for a detailed discussion. Intuitively, when simulating with a flat pricing kernel, the differences between the simulated restricted and unrestricted log scores are larger than the differences when simulating with a decreasing pricing kernel. Hence, the difference we obtain from the data has to be much larger in order to reject the null hypothesis, making the risk-neutral version of the test the most conservative way to test pricing kernel monotonicity.
} 


\subsection{Alternative test statistics}

The average log score statistic is not the only way to estimate pricing kernels and assess if realized future returns were indeed drawn from a particular subjective density. We next present parametric and non-parametric alternatives to the log score, which are based on the probability integral transformation: if we knew the true subjective distribution, we could, for each realized return, look up its percentile, i.e., the cumulative probability of a return being lower or equal to the realized return. This cumulative probability will always lie between zero and one and is iid uniformly distributed. More formally, given a sequence of realized returns $r_{t}$ stemming from the true subjective density $p_{t}\left(R_{t}\right)$, the following sequence of random variables $u_{t}$ is $i$ id uniform on the unit interval:

$$
u_{t}=\int_{0}^{r_{t}} p_{t}(x) d x, \quad t=1, \ldots, N
$$

Using Eq. (3), we can substitute the subjective density $p_{t}$ with the ratio of the estimated risk-neutral density $\widehat{q}_{t}$ and the tentative pricing kernel $\widetilde{m}$ and obtain:

$$
\tilde{u}_{t}=\int_{0}^{r_{t}}\left\{\frac{\widehat{q}_{t}(x)}{\widetilde{m}(x ; \Theta)} / \int_{0}^{\infty} \frac{\widehat{q}_{t}\left(R_{t}\right)}{\widetilde{m}\left(R_{t} ; \Theta\right)} d R_{t}\right\} d x
$$

Hence, as an alternative to maximizing the average log score, we can estimate a pricing kernel by minimizing the value of any test statistic having uniformity as the null. A number of tests are available to test for uniformity of $\tilde{u}_{t}$ but only Berkowitz (2001) simultaneously tests for independence, and so we start there.

- The Berkowitz (2001) test

The first step is yet another transformation of the iid uniformly distributed $\tilde{u}_{t}$ to normality:

$$
\widetilde{z}_{t}=\Phi^{-1}\left(\widetilde{u}_{t}\right)
$$

where $\Phi$ denotes the standard normal cumulative distribution. $\widetilde{z_{t}}$ is distributed iid standard normal if $\widetilde{u}_{t}$ is iid uniform. The second step is the estimation of the following $\mathrm{AR}(1)$ regression:

$$
\tilde{z}_{t}-\mu=\rho\left(\widetilde{z}_{t-1}-\mu\right)+\epsilon_{t}
$$

where $\epsilon_{t} \sim \mathcal{N}(0,1)$. If $\widetilde{z}_{t}$ is distributed iid standard normal, then $\mu=0, \sigma=1$, $\rho=0$, and $\epsilon_{t}$ would coincide with $\widetilde{z_{t}}$. Berkowitz (2001) proposes the following likelihood ratio based test which accounts for both, iid and normality:

$$
L R_{3}=-2(L L(0,1,0)-L L(\widehat{\mu}, \widehat{\sigma}, \widehat{\rho})),
$$

where $L L(\mu, \sigma, \rho)$ denotes the log likelihood of Eq. (10).

- The Knüppel (2015) test

The Knüppel (2015) test fits the empirical moments of the uniform variables $\tilde{u}_{t}$ to their theoretical counterpart in a generalized method of moments (GMM) fashion. 
When considering $L$ moments, the test statistic is given by

$$
\widetilde{\alpha}_{L}=N \cdot \widetilde{D}_{L}^{\prime} \widetilde{\Omega}_{L}^{-1} \widetilde{D}_{L}
$$

Here, $\widetilde{D}_{L}$ is a vector of length $L$ consisting of the differences between sample moments and their theoretical values under the null of uniformity

$$
\widetilde{D}_{L}=\left(\begin{array}{cc}
\frac{1}{N} \sum_{t=1}^{N} \tilde{u}_{t}^{1}-E\left[u^{1}\right] \\
\frac{1}{N} \sum_{t=1}^{N} \widetilde{u}_{t}^{2}-E\left[u^{2}\right] \\
\vdots \\
\frac{1}{N} \sum_{t=1}^{N} \tilde{u}_{t}^{L}-E\left[u^{L}\right]
\end{array}\right)
$$

Furthermore, $\widetilde{\Omega}_{L}$ is a consistent estimator of the covariance matrix of

$$
\tilde{d}_{t}=\left(\begin{array}{c}
\widetilde{u}_{t}^{1}-E\left[u^{1}\right] \\
\widetilde{u}_{t}^{2}-E\left[u^{2}\right] \\
\vdots \\
\widetilde{u}_{t}^{L}-E\left[u^{L}\right]
\end{array}\right) .
$$

In our base procedure, following Knüppel (2015), we use the first four moments ( $L=4$ ) and set those elements of the covariance matrix to zero, which correspond to covariances between odd and even moments. We account for possible time dependence in the $\tilde{u}_{t}$ by using the Newey-West covariance matrix with the automatic lag length selection proposed by Andrews (1991). Our findings do not change when using predefined lag lengths or a quadratic spectral covariance matrix.

- The Cramér van Mises test ${ }^{14}$

In contrast, the Cramér van Mises statistic cannot account for dependence in $\widetilde{u}_{t}$. It tests the entire distribution instead of the first central moments by comparing the empirical cumulative distribution of a sample to its theoretical counterpart. Being non-parametric, the test has less power than a parametric test such as Berkowitz (2001). The test statistic is:

$$
C v M=\int_{0}^{1}(\widehat{F}(u)-u)^{2} d u
$$

where $\widehat{F}$ denotes the empirical cumulative density function of the $\widehat{u}_{t}$ series.

We choose these statistics for a number of reasons. For one, we like to use the same criteria as the earlier studies: The Berkowitz (2001) test is the main test in Bliss and Panigirtzoglou (2004). Linn et al. (2017) fit the raw moments of the uniform distribution, similarly to the Knüppel (2015) test, in a first model selection step and apply distributional tests such as the Cramér van Mises statistic afterwards to comment on the fit, given a fixed number of moment conditions. Results based on the

14 See Cramer (1928). 
Kolmogorov-Smirnov test or relative entropy are very similar to the ones from the Cramér van Mises statistic and are not reported. The Knüppel (2015) test is chosen as it provides a reasonable trade-off between power and precision: While the Berkowitz (2001) test is only able to detect deviations from non-normality through the first and second moments, the Cramér van Mises test, examining the entire distribution, has far less power than the Berkowitz (2001) test. By using the Knüppel (2015) test with four moments, we are able to detect higher moment differences and still have enough power to test our hypothesis.

\section{Data}

As the power of the previously mentioned test statistics benefits from large sample sizes, we choose the maximal reasonable time span: data on the European options on the S\&P 500 index from January 1988 to August 2015. The data from 1988 to 1995 stem from the Berkeley Options Database, while the data from 1996 to 2015 are provided by OptionMetrics. Although the Berkeley database provides option quotes prior to 1988 , we drop these data as there is evidence that the pricing kernel puzzle did not exist in the S\&P 500 data before the crash of 1987, see Jackwerth (2000). Both databases also contain the corresponding return series for the underlying as well as dividends and the risk-free rate. We apply the usual filters to the option data insofar as options with moneyness exceeding 1.2 and below 0.8 are excluded from the sample, as well as option prices violating general no-arbitrage constraints, and quotes without volume. Consistent with the literature, we only use out-of-the-money calls and puts and consider a monthly horizon in order to obtain non-overlapping returns, which typically leads to a maturity of 23 calendar days. We end up using $N=333$ nonoverlapping monthly risk-neutral densities $\widehat{q}_{t}$ and realized returns $R_{t}$. In robustness tests, we alternatively use non-overlapping two- and three-month returns. ${ }^{15}$ For the risk-neutral densities, we report in Table 1, Panel A, the annualized average expected return $(1.29 \%)$ and the annualized average volatility $(18.57 \%)$ for the full sample. In Panel B, we report the annualized sample average (11.15\%) and the sample standard deviation (15.49\%) of the realized returns. These values are consistent with a positive equity risk-premium and a negative variance risk-premium. The annualized average risk-free rate $(3.68 \%)$ is slightly above the average dividend yield $(2.28 \%)$, which is in line with the low average expected return of the risk-neutral densities.

\section{Empirical findings}

We next present the empirical findings leading to the conclusion that the pricing kernel puzzle exists in the S\&P 500 data, even though we use forward looking information

\footnotetext{
15 Theoretically, one could also consider overlapping returns, e.g. monthly returns shifted by on day at a time. While the estimation of the unrestricted and restricted pricing kernel would be no issue in such setting, the simulation of the $p$ values of the $\Delta$ statistic is no longer straightforward. One would have to draw overlapping returns stemming from daily non-parametric densities with a monthly horizon. It is not clear at all how to do this without making restrictive assumptions on the data generating process.
} 
Table 1 Descriptive statistics

\begin{tabular}{lcc}
\hline & Average expected return (\%) & Average volatility (\%) \\
\hline $\begin{array}{l}\text { Panel A } \\
\text { Risk-neutral }\end{array}$ & 1.29 & 18.57 \\
\hline \multicolumn{2}{c}{ Sample mean (\%) } & Sample standard deviation (\%) \\
\hline $\begin{array}{l}\text { Panel B } \\
\text { Realized returns }\end{array}$ & 15.49 \\
Risk-free rate & 11.15 & 2.62 \\
Dividend yield & 3.68 & 1.20 \\
\hline $\begin{array}{l}\text { We report descriptive statistics for risk neutral densities, realized returns, risk-free rates, and dividend yields. } \\
\text { Panel A shows the annualized average of the expected returns and the average volatility of the risk-neutral } \\
\text { densities. Panel B displays sample means and standard deviations of the realized returns, the risk-free rates, } \\
\text { and the dividend yields }\end{array}$
\end{tabular}

Table 2 Results for the linear pricing kernel optimization-main table

\begin{tabular}{llllll}
\hline Statistic & Unrestricted & Restricted & $\gamma=0$ & $\gamma=2$ & $\gamma=4$ \\
\hline Log score & 2.00 & 1.98 & 0.02 & 0.01 & 0.01 \\
Knüppel & 0.00 & 9.90 & 0.02 & 0.00 & 0.00 \\
Cramér van Mises & 47.31 & 294.10 & 0.34 & 0.06 & 0.01 \\
Berkowitz & 0.57 & 24.33 & 0.98 & 0.92 & 0.82 \\
\hline
\end{tabular}

Estimation results when fitting a piecewise linear pricing kernel, with nine points in the return space and optimized values in the pricing kernel space, to the full sample. The numbers in the second and third columns show the test statistics associated with the measure mentioned in the first column. The $p$ values of the difference between the unrestricted and restricted test statistic are given in columns four to six. All $p$ values are obtained by simulation, assuming that the true pricing kernel stems from a power utility function with $\gamma=0,2$, and 4, respectively. The values of the Cramér van Mises test are multiplied by one million and the values of the Berkowitz (2001) test are multiplied by $10^{12}$

only. We first look at the statistical evidence against pricing kernel monotonicity. Afterwards, we discuss the shape of the empirical pricing kernel estimates and refer to the literature.

\subsection{Statistical evidence}

In Table 2, we show the estimation results stemming from the linear pricing kernel interpolation as described in Sect. 2.2. The values for the optimal test statistics are collected in the columns Unrestricted and Restricted for the respective pricing kernel specification. The distributions of the differences between the two specifications are simulated by the procedure described in Sect. 2.4. Based on the simulated distributions, we report in the remaining columns of Table $2 p$ values corresponding to the differences, assuming that the true pricing kernel is non-increasing and stems from a power utility function with $\gamma=0, \gamma=2$, and $\gamma=4$, respectively. 
Considering the average log score in the most conservative setting with $\gamma=0$, we reject the null hypothesis of a non-increasing pricing kernel ( $p$ value $2 \%$ ). The Knüppel (2015) test confirms this finding ( $p$ value 2\%). The Cramér van Mises test achieves a $p$ value of $34 \%$ and cannot reject non-increasing pricing kernels, which could be due to the relatively low power of non-parametric tests compared with parametric tests. The $p$ value of the Berkowitz (2001) test is large at 0.98 . This is not surprising since this test only takes into account the mean and variance of the transformed variables, as it is based on the likelihood function of the normal distribution. Mitchell and Hall (2005) point out that the Berkowitz (2001) test only "has power to detect non-normality through the first two moments," but, e.g., excess skewness and kurtosis are not captured. We confirm this finding by running the Knüppel (2015) test with the first two moments $(L=2)$ only and find that monotonicity cannot be rejected as well. This holds true irrespective of taking care of serial correlation by a proper covariance matrix $\widetilde{\Omega}_{L}$ or focusing on the moments only; i.e. basing the covariance matrix on the identity matrix. Even though we are aware of the drawbacks of the Berkowitz (2001) test, we report the corresponding results as it is the main test used in Bliss and Panigirtzoglou (2004), which was the first study to back out the pricing kernel from forward looking option prices only.

Before launching into the discussion of the results for the risk averse settings ( $\gamma$ being either 2 or 4), some words of caution are in order. Rejection in such setting is only a rejection of the null hypothesis that the true pricing kernel in the economy derives from a power utility function with the stated risk aversion coefficient. The tests cannot detect monotonicity violations for other utility functions or power utilities with other risk aversion coefficients. Note that this critique does not hold for the case of risk neutrality $(\gamma=0)$, which is the most conservative test for general pricing kernel monotonicity.

We still feel that the risk averse settings are interesting as they are standard reference points for economists. Recall that Bliss and Panigirtzoglou (2004) found $\gamma=4.08$ as the optimal relative risk-aversion parameter for a power utility when looking at a monthly horizon. With risk-aversion, all $p$ values are below $10 \%$, except for the Berkowitz (2001) test with $p$ values of more than $80 \%$. Note that we obtain similar results for pricing kernels based on exponential utility functions. ${ }^{16}$

Overall, for all tests but the Berkowitz (2001) test, the difference between an unrestricted and a restricted pricing kernel is significantly different from zero, and, therefore, we reject the hypothesis of a non-increasing pricing kernel. The Cramér van Mises test does not reject monotonicity in the $\gamma=0$ setting as, being non-parametric, its power is too limited. The parametric Berkowitz (2001) test, which would be wellsuited for small samples in terms of power, ignores moments higher than the second and also fails to reject.

\footnotetext{
16 The pricing kernel derived from an exponential utility function is given by $m^{\exp .}=\exp \left(-a \cdot R_{t}\right)$. Bliss and Panigirtzoglou (2004) identified $a=6.33$ as the optimal value when looking at a monthly horizon and so we repeated our base procedure for $a=2,4,6,8$, and 10. Again, all $p$ values decrease in $a$. All our tests, except the Berkowitz (2001) test, reject pricing kernel monotonicity at least at the $10 \%$ level.
} 

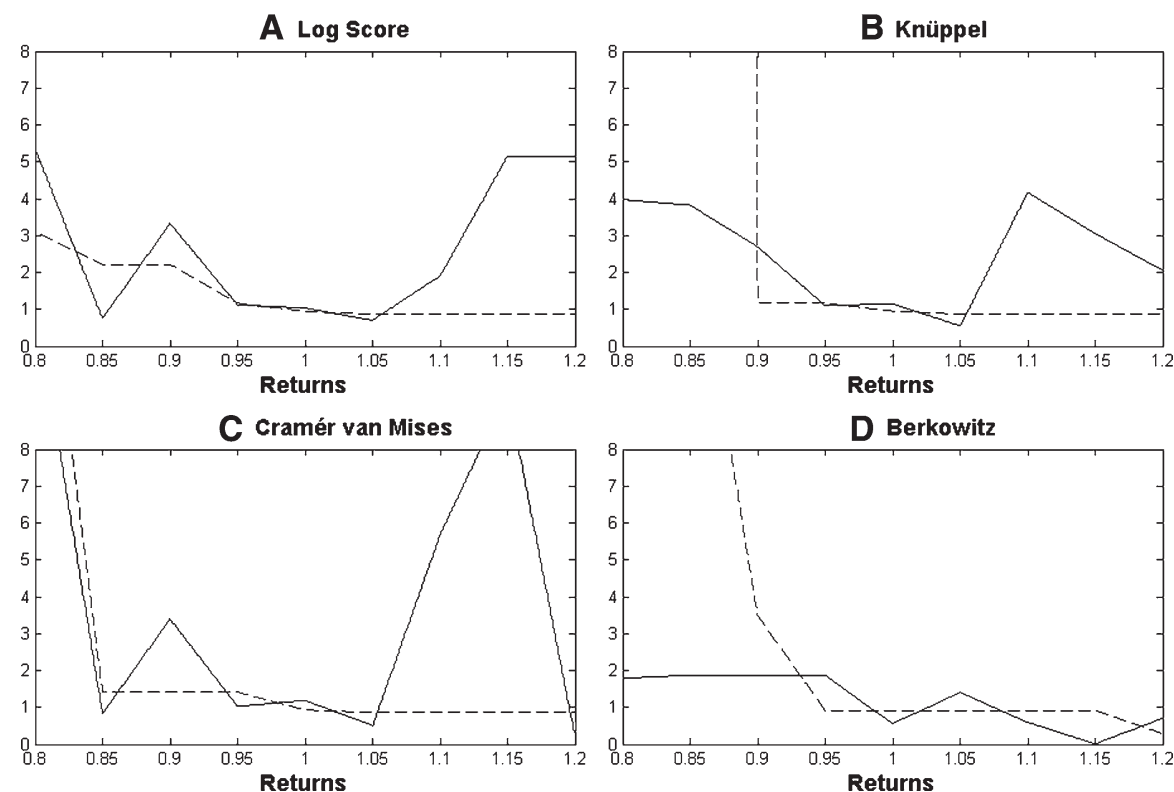

Fig. 2 Optimal pricing kernels for different measures. We plot unrestricted scaled pricing kernels (solid) and a non-increasing scaled pricing kernels (dotted), constructed by our linear interpolation rule on the full sample. All pricing kernels are scaled by the risk-neutral density from 28th January 2015. Panel A shows the pricing kernels when maximizing the average log score. Panel B shows the pricing kernels when minimizing the Knüppel test statistic. Similarly for Panel C and D for the Cramér van Mises and the Berkowitz test statistics. Returns are on the horizontal axis, and the values of the pricing kernel are on the vertical axis

\subsection{The shape of the empirical pricing kernels}

Having documented that pricing kernel monotonicity can be rejected in most reasonable settings, we turn our attention to the shape of the empirical pricing kernels. Panel A of Fig. 2 repeats Fig. 1 and shows the optimal unrestricted and restricted pricing kernel estimates when maximizing the average log score. The optimal pricing kernels from minimizing the Knüppel (2015), Cramér van Mises, and Berkowitz (2001) test statistics are depicted in Panels B, C, and D of Fig. 2, respectively. The overall shapes of the empirical pricing kernels are robust to variations in the methodology, see Sects. 5.2 and 5.3.

Except for optimizing the Berkowitz (2001) test, all empirical pricing kernels are broadly u-shaped, consistent with the parametric option pricing model of Christoffersen et al. (2013) where past returns are used for inferring the subjective density. Using past returns for inferring the subjective density, Cuesdeanu (2016) finds that the pricing kernel is strictly $\mathrm{u}$-shaped when the variance-risk-premium is high and wshaped when the variance-risk-premium is low. Furthermore, not finding either u- or w-shaped pricing kernels seems due to data limitations and the time-series model used 
for obtaining the subjective density. ${ }^{17}$ Hence, when interpreting our unrestricted pricing kernels as average pricing kernels over time, it is not surprising that they display a w-shape, similar to the ones in Cuesdeanu (2016). ${ }^{18}$

Bakshi et al. (2010), Polkovnichenko and Zhao (2013), and Chabi-Yo (2012) develop theories for u-shaped pricing kernels. Bakshi et al. (2010) assume heterogeneity among investors, and pessimists shorting the market lead to increases in the pricing kernel for increasing returns larger than some threshold. Polkovnichenko and Zhao (2013) claim that investors overweight the subjective probability of high and low returns, which again yields a u-shaped pricing kernel. Finally, Chabi-Yo (2012) describes an economy where skewness and kurtosis preferences enter the pricing kernel. His empirical pricing kernel, estimated from the returns of 30 monthly industry portfolios displays a u-shape, too, when being projected on the market return. The Berkowitz (2001) test, ignoring moments higher than the second, could not reject pricing kernel monotonicity. Such failure might be related to the existence of higher moment preferences in the pricing kernel. In the next section we document that the $\mathrm{u}$-shape is very persistent and survives various robustness checks.

\section{Robustness}

We show that our results are robust to a number of changes, namely, changing the sample period, using alternative pricing kernel specifications (spline instead of piecewise linear), and employing different methods for the risk-neutral densities (Gatheral 2004 instead of Jackwerth 2004). Concerning lengthening horizons, the pricing kernel puzzle weakens for longer horizons. Finally, we investigate exactly why Linn et al. (2017) obtain monotonically decreasing pricing kernels.

\subsection{Alternative sub-samples}

An important issue is the choice of the sample period. As there is no evidence for the pricing kernel puzzle in the S\&P 500 data before the crash of 1987, we choose January 1988 as our starting date. The largest available sample then extends until August 2015 and is used in the base case. A large sample makes our study more reliable from a statistical point of view, but the assumption of time invariant preferences might be tenuous over a period of almost 30 years. As volatility is considered as a relevant state variable for preferences, see e.g. Brown and Jackwerth (2012), we follow Bliss and Panigirtzoglou (2004) by dividing our sample into a high and a low volatility

17 In particular, Cuesdeanu (2016) argues that the tilde shaped pricing kernels in the canonical papers of AitSahalia and Lo (2000), Jackwerth (2000), and Rosenberg and Engle (2002) can be explained by the sample period (characterized by a relatively low variance-risk-premium) in combination with the non-observability of out-of-the-money calls.

18 A model that could potentially explain w-shaped pricing kernels in returns is Bakshi et al. (2015). The authors describe an economy where investors are long and short volatility, and aggregation leads to a ushaped pricing kernel in the volatility dimension. Since high (low) absolute returns are typically associated with high (low) volatility, a u-shaped pricing kernel in the volatility dimension could mechanically imply a w-shape in the return dimension. 
Table 3 Alternative sub-samples

\begin{tabular}{llllll}
\hline Statistic & Basic setting & High vol & Low vol & $1996-2012$ & $1996-2015$ \\
\hline Panel $A: \gamma=0$ & & & & & \\
Log score & 0.02 & 0.03 & 0.26 & 0.01 & 0.01 \\
Knüppel & 0.02 & 0.19 & 0.04 & 0.01 & 0.04 \\
Cramér van Mises & 0.34 & 0.21 & 0.25 & 0.15 & 0.31 \\
Berkowitz & 0.98 & 0.95 & 0.09 & 0.73 & 0.96 \\
Panel B: $\gamma=2$ & & & & & \\
Log score & 0.01 & 0.01 & 0.16 & 0.00 & 0.00 \\
Knüppel & 0.00 & 0.06 & 0.02 & 0.00 & 0.01 \\
Cramér van Mises & 0.06 & 0.09 & 0.17 & 0.05 & 0.11 \\
Berkowitz & 0.92 & 0.85 & 0.05 & 0.43 & 0.82 \\
Panel $C: \gamma=4$ & & & & & \\
Log score & 0.01 & 0.01 & 0.12 & 0.00 & 0.00 \\
Knüppel & 0.00 & 0.03 & 0.01 & 0.00 & 0.00 \\
Cramér van Mises & 0.01 & 0.03 & 0.09 & 0.01 & 0.03 \\
Berkowitz & 0.82 & 0.77 & 0.03 & 0.33 & 0.68 \\
\hline Estimating
\end{tabular}

Estimation results when fitting a piecewise linear pricing kernel, with nine points in the return space and optimized values in the pricing kernel space, to alternative samples. We show the $p$ values of the difference between the unrestricted and restricted test statistics mentioned in the first column. We simulate all $p$ values, assuming that the true pricing kernel stems from a power utility function with $\gamma=0$ in Panel A, $\gamma=2$ in Panel B, and $\gamma=4$ in Panel C. Column 2 of each panel contains the basic setting from Table 2. In the other columns, we use different samples: 'High Vol' uses all days with above median volatility, and 'Low Vol' uses the remaining days. '1996-2012' and '1996-2015' use the respective years

subsample. The dividing line is the median of the monthly risk-neutral volatilities from 1988 to 2015. We report the results in Table 3, columns 'High Vol' and 'Low Vol'. We simulate all $p$ values using the procedure described in Sect. 2.4. Panels A, $\mathrm{B}$, and $\mathrm{C}$ correspond to the settings where we assume that the true pricing kernel is non-increasing and stems from a power utility function with $\gamma=0,2$, and 4 , respectively.

For most settings in the high volatility sample, we still reject the null of a nonincreasing pricing kernel, see Table 3, 'High Vol'. Compared to the base setting, only the Knüppel (2015) test now fails to reject monotonicity with a $p$ value of $19 \%$ in the risk-neutral simulation setting. For the low volatility sample (see Table 3, 'Low Vol'), the $\log$ score and the Cramér van Mises test turn insignificant, while the Knüppel (2015) test holds up well. Interestingly, the Berkowitz (2001) test is able to reject pricing kernel monotonicity in the low volatility sample with a $p$ value of $9 \%$ for $\gamma=0$ and even stronger results for higher $\gamma \cdot{ }^{19}$

Another way of relaxing the assumption on time invariant preferences is to calibrate our model to the sub-sample used by Linn et al. (2017): September 1996 to December

19 The Berkowitz (2001) test might gain in power as the higher moments of the returns realized in this subsample are less extreme. Compared to the full sample, sample kurtosis drops from 7.18 to 3.74 and sample skewness is less negative: -1.24 in the full sample versus -0.66 in the low volatility subsample. 

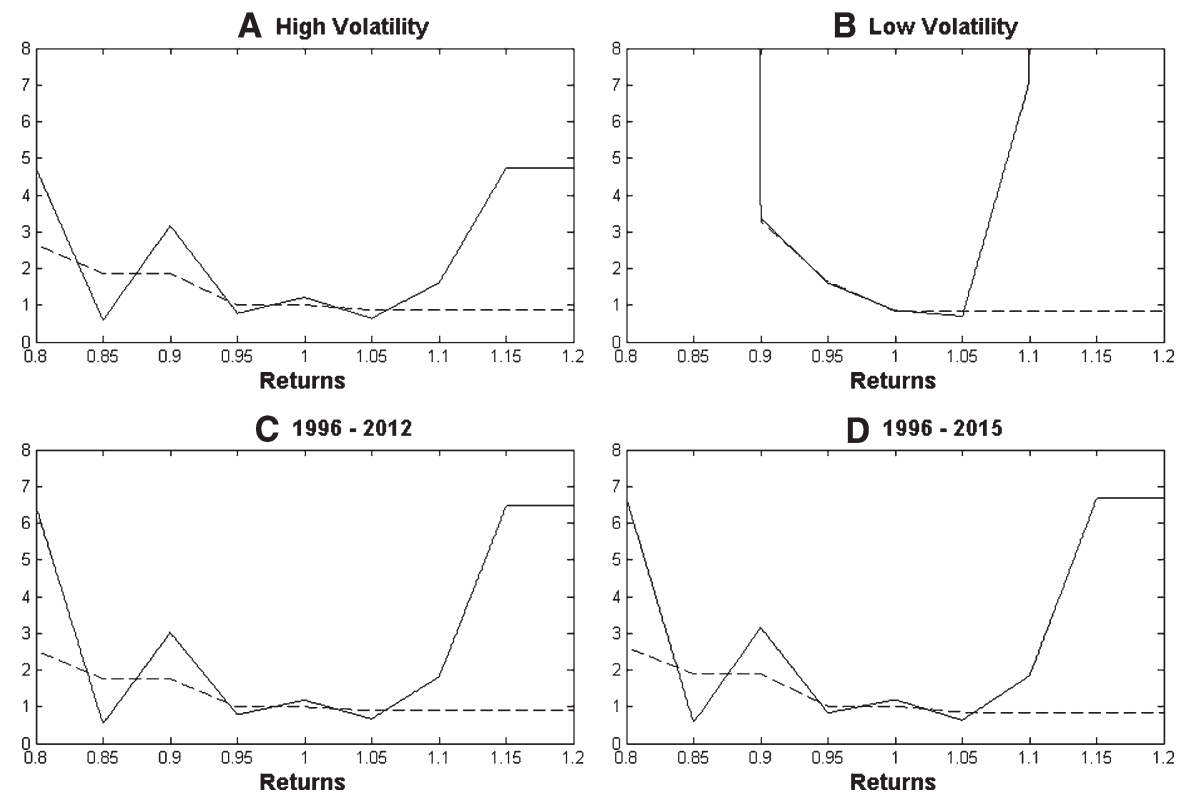

Fig. 3 Optimal pricing kernels on different sub-samples. We plot unrestricted scaled pricing kernels (solid) and a non-increasing scaled pricing kernels (dotted), constructed by our linear interpolation rule and maximizing the average log score as given in Eq. (4). All pricing kernels are scaled by the risk-neutral density from 28th January 2015. The Panels A and B show the pricing kernels estimated on the high and low volatility samples. Panel C shows the pricing kernels estimated on the January 1996 - December 2012 sample; Panel D shows the longer sample until August 2015. Returns are on the horizontal axis, and the values of the pricing kernel are on the vertical axis

2012, so that we can relate our results to their claim of monotonically decreasing pricing kernels. The results are reported in Table 3, '1996-2012'. Still, we reject non-increasing pricing kernels at the $1 \%$ level for the log score and the Knüppel (2015) test. The Cramér van Mises test even improves in this sample and generates a marginally insignificant $p$ value of $15 \%$ in the $\gamma=0$ setting. We return to the reasons for our disagreement with Linn et al. (2017) in Sect. 5.5. The results for the whole OptionMetrics sample in Table 3, '1999-2015', are rather similar.

In conclusion, monotonicity violations seem to be present in all subsamples. The moderate increases in $p$ values could well be due to the smaller subsamples. In Fig. 3 , we depict the optimal pricing kernels (based on the log score) for our subsamples. Their shapes hardly change compared to the full sample, depicted in Fig. 1, except for the low volatility case, where the unrestricted pricing kernel is even more u-shaped.

\subsection{Alternative pricing kernel modeling}

As most theoretical pricing kernels are assumed to be smooth functions and to mimic the methodology of Linn et al. (2017) even more closely, we replace the linear interpolation of the pricing kernel with cubic splines. To guarantee the non-negativity of all constructed pricing kernels, we first interpolate the logarithm of the eligible pric- 
Table 4 Alternative modeling

\begin{tabular}{llllll}
\hline Statistic & Basic setting & Cubic spline & 5 Points & Different grid & Gatheral SVI \\
\hline Panel $A: \gamma=0$ & & & & & \\
Log score & 0.02 & 0.02 & 0.01 & 0.12 & 0.02 \\
Knüppel & 0.02 & 0.09 & 0.01 & 0.04 & 0.03 \\
Cramér van Mises & 0.34 & 0.38 & 0.27 & 0.46 & 0.39 \\
Berkowitz & 0.98 & 0.99 & 0.95 & 0.99 & 0.93 \\
Panel B: $\gamma=2$ & & & & & \\
Log score & 0.01 & 0.00 & 0.00 & 0.04 & 0.00 \\
Knüppel & 0.00 & 0.02 & 0.00 & 0.01 & 0.01 \\
Cramér van Mises & 0.06 & 0.08 & 0.06 & 0.14 & 0.08 \\
Berkowitz & 0.92 & 0.93 & 0.83 & 0.96 & 0.74 \\
Panel C: $\gamma=4$ & & & & & \\
Log score & 0.01 & 0.00 & 0.00 & 0.02 & 0.00 \\
Knüppel & 0.00 & 0.01 & 0.00 & 0.00 & 0.00 \\
Cramér van Mises & 0.01 & 0.01 & 0.01 & 0.03 & 0.02 \\
Berkowitz & 0.82 & 0.84 & 0.76 & 0.93 & 0.62 \\
\hline Estim ren & & & & 0.03 &
\end{tabular}

Estimation results when fitting alternative pricing kernels or risk-neutral densities obtained by the SVI model on the full sample. We show the $p$ values of the difference between the unrestricted and restricted test statistics mentioned in the first column. We simulate all $p$ values, assuming that the true pricing kernel stems from a power utility function with $\gamma=0$ in Panel A, $\gamma=2$ in Panel B, and $\gamma=4$ in Panel C. Column 2 of each panel contains the basic setting from Table 2. We present results for cubic spline interpolation instead of linearly interpolated pricing kernels ('Cubic Spline'), for pricing kernels spanned by only 5 instead of 9 points (' 5 Points'), for a shifted set of grid values on the horizontal axis ('Different Grid'), and for using the SVI method of Gatheral (2004) instead of the fast and stable method of Jackwerth (2004) ('Gatheral SVI')

ing kernel values and afterwards take the exponential of the interpolated values. The results of this calibration are given in Table 4, 'Cubic Spline'. Our findings do not change much even though the test is more conservative when using cubic splines: In order to have simple constraints for estimating the restricted version of our pricing kernels, we label a tentative pricing kernel as non-increasing whenever the values of the pricing kernel at the spline knots are non-increasing. Hence, all non-increasing pricing kernels are consistent with our definition of a restricted kernel, but a restricted kernel constructed by cubic splines can have slightly increasing segments due to the curvature of the cubic splines. ${ }^{20}$ A restricted pricing kernel can somewhat mimic the shape of an unrestricted pricing kernel and improve its fit to the respective criteria. This is for example the case when optimizing the Knüppel (2015) test: The optimal restricted cubic spline pricing kernel is slightly increasing in the right end, raising the $p$ value to $9 \%$ in the $\gamma=0$ setting. Rejection of non-increasing pricing kernels based on the cubic spline interpolation is therefore even more meaningful as we can clearly distinguish between small fluctuations and pronounced increasing segments.

20 In case of linear interpolation, a pricing kernel is non-decreasing if and only if its values at the spline knots are non-increasing. 
Our results are also not driven by over-fitting, as we can see from the results from estimating the pricing kernel with only 5 points, see Table 4, '5 Points'. Changing the location of our 9 points in the base case does not affect results, either, see Table 4, 'Different Grid'. In that case, we use the midpoints of the basic grid (0.825 0.875 0.9250 .9751 .0251 .0751 .1251 .175 ) and only the average log score turns out to be marginally insignificant with a $p$ value of $12 \%$ in the $\gamma=0$ setting.

\subsection{Alternative risk neutral density modeling}

In the base case, we extrapolate the implied volatility curve by the fast and stable method of Jackwerth (2004). As an alternative, we use the Stochastic Volatility Inspired (SVI) model of Gatheral (2004) instead. As the name already indicates, its structural form is related to models such as Heston (1993). The model is fully parameterized and does not have a smoothness parameter, such as $\lambda$ from Eq. (2) in the case of the fast and stable method, which can be chosen by the econometrician. The parameterization is as follows:

$$
\sigma_{S V I}^{2}(K \mid a, b, \rho, m, \theta)=a+b\left\{\rho\left(\log \left(K / S_{t}\right)-m\right)+\sqrt{\left(\log \left(K / S_{t}\right)-m\right)^{2}+\theta^{2}}\right\}
$$

Here, $a$ is the average squared implied volatility and has to be positive. The nonnegative parameter $b$ measures the angle between the slope of in-the-money to out-ofthe-money volatilities. $\rho$ and $m$ are location parameters, and $\theta$ controls the curvature around at-the-money. In order to fit the implied volatility curve (16) to the data, we numerically minimize the squared difference between model implied volatilities and implied volatilities observed in the market:

$$
\left\{a^{*}, b^{*}, \rho^{*}, m^{*}, \theta^{*}\right\}=\underset{\{a, b, \rho, m, \theta\}}{\arg \min } \sum_{i=1}^{I}\left(\sigma_{S V I}\left(K_{i} \mid a, b, \rho, m, \theta\right)-\bar{\sigma}_{i}\right)^{2} .
$$

The smooth implied volatility curve is given by

$$
\left\{\sigma_{S V I}\left(K_{j} \mid a^{*}, b^{*}, \rho^{*}, m^{*}, \theta^{*}\right)\right\}_{j=0}^{J},
$$

and the risk-neutral densities are again obtained by means of Breeden and Litzenberger (1978).

In Table 4, 'Gatheral SVI', we report the results for a piece-wise linear pricing kernel and risk-neutral densities stemming from the SVI model. Again, assuming risk-aversion, non-increasing pricing kernels can be rejected at the $10 \%$ level with the exception of the Berkowitz (2001) test. For the conservative version with $\gamma=0$, the Cramér van Mises statistic fails to reject with a $p$ value of $39 \%$, similar to the base case when using the fast and stable method. 
Table 5 Different horizons

\begin{tabular}{|c|c|c|c|c|c|c|}
\hline \multirow[t]{2}{*}{ Statistic } & \multirow{2}{*}{$\begin{array}{l}\text { Basic } \\
\text { Setting }\end{array}$} & \multicolumn{2}{|c|}{2 Months } & \multicolumn{3}{|c|}{3 Months } \\
\hline & & Jan & Feb & Jan & Feb & Mar \\
\hline Log score & 0.01 & 0.13 & 0.14 & 0.06 & 0.13 & 0.43 \\
\hline Knüppel & 0.00 & 0.07 & 0.17 & 0.16 & 0.30 & 0.17 \\
\hline Cramér van Mises & 0.01 & 0.09 & 0.17 & 0.18 & 0.14 & 0.20 \\
\hline Berkowitz & 0.82 & 0.39 & 0.02 & 0.04 & 0.03 & 0.11 \\
\hline
\end{tabular}

Estimation results when fitting a piecewise linear pricing kernel, with nine points in the return space and optimized values in the pricing kernel space, on different horizons. We show $p$ values of the difference between the unrestricted and restricted test statistics mentioned in the first column. Column 2 contains the basic setting from Table 2. We report the results for non-overlapping two months returns in the columns '2 Months, Jan' and '2 Month, Feb'. We report the results for non-overlapping three months returns in the columns '3 Months, Jan', '3 Months, Feb', and '3 Months, Mar'. We simulate all $p$ values, assuming that the true pricing kernel stems from a power utility function with $\gamma=4$

\subsection{Different horizons}

The pricing kernel puzzle arises from the different shapes of subjective and risk-neutral distributions as the former tend to be more (log-)normally shaped while the latter are heavily leptokurtic and left-skewed. At longer horizons, the risk-neutral distributions look more (log-)normally shaped and we thus suspect that the pricing kernel puzzle might be weaker.

To check, we repeat our base procedure for horizons of two months, $T=51$, and three months, $T=79$. As we want to stick to non-overlapping returns, we can choose either January or February as the starting months when looking at a two-month horizon. For the three months setting, we can choose between January, February, and March as the starting months. This results in two sets of $N=164$ and three sets of $N=95$ non-overlapping risk-neutral densities and their corresponding non-overlapping twomonth and three-month returns. Table 5 displays the corresponding results for the case of $\gamma=4$. As expected, monotonicity is the harder to reject, the longer the considered horizon is. We do not report the results for lower $\gamma$ values, as all $p$ values tend to increase in that case; further weakening the results. For $\gamma=0$, all $p$ values are larger than $10 \%$.

We conclude that the pronounced skew of the short term implied volatilities of S\&P 500 index options, inducing left-skewness and leptokurtosis, paired with the time series properties of the monthly returns, causes the non-decreasing pricing kernel estimates at the short, monthly horizon. The pricing kernel puzzle is much attenuated at the longer two-month horizon and hardly detectable at the three-month horizon.

\subsection{Degenerate densities}

Last, we are interested in why Linn et al. (2017) obtain a monotonically decreasing pricing kernel. We apply two methodological changes which we believe cause their results. First, Linn et al. (2017) do not force the resulting subjective densities to 


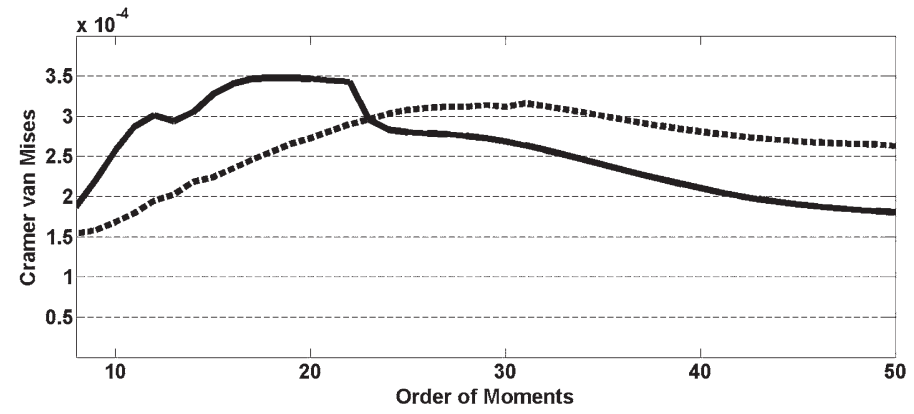

Fig. 4 Moment fit and Cramér van Mises statistic. We estimate pricing kernels by fitting the first $L$ moments of the uniform distribution without scaling the resulting subjective densities. For each resulting pricing kernel, we calculate the corresponding Cramér van Mises statistic. The Cramér van Mises values stemming from the unrestricted (restricted) pricing kernels are plotted with a solid (dashed) line. The number of moment conditions $L$ is on the horizontal axis

integrate to one; we follow them by no longer scaling the subjective density. Second, they utilize a two-step model selection procedure: The first step consists of fitting the pricing kernel to the first $L$ moments of the uniform distribution by minimizing the following objective function ${ }^{21}$

$$
G M M(L)=\sum_{l=1}^{L}\left(\frac{1}{N} \sum_{t=1}^{N} \widetilde{u}_{t}^{l}-\frac{1}{l+1}\right)^{2}
$$

This optimization is implemented for up to $L=50$ moment conditions, providing a variety of potential pricing kernels. ${ }^{22}$ For each of these pricing kernels, the authors compute the Cramér van Mises statistic, and the second model selection step consists of choosing the pricing kernel delivering the lowest test statistic.

We again mimic their procedure with our linear pricing kernels without scaling the subjective densities, and we depict the relationship between the number of fitted moments $L$ and the associated Cramér van Mises statistic in Fig. 4. We also follow Linn et al. (2017) by ignoring the fit to moments of order smaller than the number of pricing kernel parameters as, according to the authors, this leads to an under-identified GMM equation system. As our linear pricing kernel is a function of eight parameters, we start at $L=8$ and fit unrestricted (solid line) as well as restricted (dashed line) pricing kernels to our full sample, leaving us with $86(=43 \cdot 2)$ potential estimates.

21 This GMM approach is equivalent to setting $\widetilde{\Omega}_{L}$ to the identity matrix in Knüppel (2015).

22 Note that Linn et al. (2017) discuss the results for all numbers of moment conditions up to $L=50$ in the working paper version only. In the published version of their paper they only show the results for $L \in\{4,5,6,7,8,9\}$ and claim that the best pricing kernel obtains when the number of pricing kernel parameters equals to the number of moment conditions. They find that for the S\&P 500, choosing $L=7$ delivers the highest $p$ values for the Berkowitz, Cramér van Mises, and Kolmogorov-Smirnov test statistics. Note that the $p$ values in their Table 2 are upwards biased since fitting the moments of a distribution helps in fitting the distribution itself. Only the $p$ values corresponding to the rows labelled 'No pricing kernel' are unbiased since no pricing kernel optimization happened there. 
Regarding Fig. 4, it is not only surprising that the overall minimum is obtained by a restricted pricing kernel for $L=8$, but the restricted versions tend to provide better Cramér van Mises statistics for $L$ less than 23. This is surprising as one an unrestricted optimum should perform at least as well than a restricted optimum. But, by ignoring the scaling of the subjective densities and using the two-step model selection, which mixes two different measures of fit, a monotonically decreasing pricing kernel can be identified as the best forward looking pricing kernel estimate, just as in Linn et al. (2017).

Looking back at the integral of Eq. (8), we can see why Linn et al. (2017) might not want to scale the subjective densities: In order to obtain the transformed realized returns series $\widetilde{u}_{t}$, one has to evaluate the integrand only up to the maximal realized return in the sample. ${ }^{23}$ The advantage of their approach is that they do not have to model the pricing kernel for returns where no realized return is observed. Moreover, their pricing kernel is literally constant through time, whereas our pricing kernel is scaled by a time varying factor.

However, the two modifications have to be seen critically: not scaling the subjective densities clashes with our intuition that probabilities should sum up to one and can make the $\tilde{u}_{t}$ series lie outside the unit interval. Moreover, for consistency, the same loss function should be used for estimating and evaluating a model, see Christoffersen and Jacobs (2004), which speaks against their two-step procedure of GMM and Cramér van Mises in combination.

We want to emphasize that only the combination of not scaling the subjective densities and the two-step procedure leads to a monotonically decreasing pricing kernel. First, when replicating the results shown in Fig. 4 with scaled subjective densities, the surprising finding disappears: For any number $L$ of moment conditions, the unrestricted pricing kernel is now delivering a lower Cramér van Mises statistic than the restricted pricing kernel, i.e., the solid line is always below the dotted line. Second, when leaving the subjective densities unscaled but minimizing the GMM objective function, an unrestricted pricing kernel again delivers a lower GMM value than a restricted kernel. The same holds if we use only the Cramér van Mises statistic instead.

In conclusion, both, the two-step procedure with scaled subjective densities and a single objective function minimization with unscaled subjective densities would identify a non-monotonically decreasing pricing kernel as the best estimate. This is in line with the results of our own procedure, when optimizing a single criteria and scaling the subjective densities.

\section{Conclusion}

By using forward looking information only (i.e., option prices and future realized returns), we confirm the existence of the pricing kernel puzzle in the S\&P 500 data. This contradicts the results in Linn et al. (2017), which we attribute to a lack of scaling the resulting subjective densities and a particular choice of two-step optimization in their paper. Potential pricing kernels are modeled as piece-wise linear functions and

23 The maximal realized return in our sample is at about 1.11 . 
cubic splines. Forward looking subjective densities are constructed by dividing the forward looking risk-neutral density by a time invariant pricing kernel. We make use of the average log score, as well as three alternative distributional tests, to asses if the forward looking subjective densities are compatible with the future realized returns of the S\&P 500. By varying the potential pricing kernel, we can optimize the unrestricted test statistics. We also optimize the test statistics in a restricted version, where the pricing kernel needs to be non-increasing.

We formally test for monotonicity based on the difference between the unrestricted and the restricted test statistics. The average log score and the Knüppel (2015) test reject pricing kernel monotonicity with a $p$ value of $2 \%$. Non-rejection with the latter two criteria occurs only very rarely for subsamples. The Berkowitz (2001) test is the only test that does not reject non-increasing pricing kernels. This tests takes into account only the first two moments and ignores all higher moments. Yet, the importance of non-normal skewness and kurtosis is well documented in the financial time series literature. Due to its low power in small samples, the Cramér van Mises statistic does not reject pricing kernel monotonicity in the most conservative setting, where we simulate a risk-neutral economy.

Overall, our results are robust to variations in the methodology and the sample. We show that pricing kernel monotonicity cannot be rejected when looking at longer horizons, where subjective and risk-neutral densities are less negatively skewed and less kurtotic. Our findings suggest that solutions to the pricing kernel puzzle should therefore consider higher moment preferences.

\section{References}

Ait-Sahalia, Y., \& Lo, A. W. (2000). Nonparametric risk management and implied risk aversion. Journal of Econometrics, 94, 9-51.

Andrews, D. W. K. (1991). Heteroskedasticity and autocorrelation consistent covariance matrix estimation. Econometrica, 59, 817-858.

Babaoglu, K., Christoffersen, P., Heston, S., \& Jacobs, K. (2017). Option valuation with volatility components, fat tails, and nonlinear pricing kernels. Review of Asset Pricing Studies, forthcoming.

Bakshi, G., Madan, D. B., \& Panayotov, G. (2010). Returns of claims on the upside and the viability of U-shaped pricing kernels. Journal of Financial Economics, 97, 130-157.

Bakshi, G., Madan, D. B., \& Panayotov, G. (2015). Heterogeneity in beliefs and volatility tail behavior. Journal of Financial and Quantitative Analysis, 50, 1389-1414.

Barone-Adesi, G., Dall'O, H., \& Vovchak, V. (2012). Is the pricing kernel monotone? Journal of Finance and Risk Perspectives, 1, 43-69.

Barone-Adesi, G., Engle, R. F., \& Mancini, L. (2008). A GARCH option pricing model with filtered historical simulation. Review of Financial Studies, 21, 1223-1258.

Beare, B., \& Moon, J.-M. (2015). Nonparametric tests of density ratio ordering. Econometric Theory, 31, $471-492$.

Beare, B., \& Schmidt, L. (2014a). Empirical implications of the pricing kernel puzzle for the return on contingent claims. In Working paper, University of California San Diego.

Beare, B., \& Schmidt, L. (2014b). An empirical test of pricing kernel monotonicity. Journal of Applied Econometrics, 31, 338-356.

Beare, B. K. (2011). Measure preserving derivatives and the pricing kernel puzzle. Journal of Mathematical Economics, 47, 689-697.

Benzoni, L., Dufresne, C. P., \& Goldstein, R. S. (2011). Explaining asset pricing puzzles associated with the 1987 market crash. Journal of Financial Economics, 101, 552-573. 
Berkowitz, J. (2001). Testing density forecasts with applications to risk management. Journal of Business and Economic Statistics, 19, 465-474.

Bliss, R. R., \& Panigirtzoglou, N. (2004). Option-implied risk aversion estimates. Journal of Finance, 59, 407-446.

Breeden, D. T., \& Litzenberger, R. H. (1978). Prices of state-contingent claims implicit in option prices. Journal of Business, 51, 621-651.

Brown, D. P., \& Jackwerth, J. C. (2012). The pricing kernel puzzle: reconciling index option data and economic theory. Contemporary Studies in Economic and Financial Analysis, 94, 155-183.

Chabi-Yo, F. (2012). Pricing kernels with stochastic skewness and volatility risk. Management Science, 58, 624-640.

Chabi-Yo, F., Garcia, R., \& Renault, E. (2009). State dependence can explain the risk aversion puzzle. Review of Financial Studies, 21, 973-1011.

Chaudhuri, R., \& Schroder, M. D. (2015). Monotonicity of the stochastic discount factor and expected option returns. Review of Financial Studies, 28, 1462-1505.

Christoffersen, P., Heston, S., \& Jacobs, K. (2013). Capturing option anomalies with a variance-dependent pricing kernel. Review of Financial Studies, 26, 1962-2006.

Christoffersen, P., \& Jacobs, K. (2004). The importance of the loss function in option valuation. Journal of Financial Economics, 72, 291-318.

Cochrane, J. H. (2000). Asset pricing (1st ed.). Princeton, USA: Princeton University Press.

Cramer, H. (1928). On the composition of elementary errors. Scandinavian Actuarial Journal, 59, 13-74.

Cuesdeanu, H. (2016). Empirical pricing kernels: A tale of two tails and volatility? In Working paper, University of Konstanz.

Cuesdeanu, H., \& Jackwerth, J. C. (2017). The pricing kernel puzzle: Survey and outlook. Annals of Finance, forthcoming.

de Boor, C. (1978). A practical guide to splines (1st ed.). New York: Springer.

Dybvig, P. H. (1988). Distributional analysis of portfolio choice. Journal of Business, 63, 369-393.

Gatheral, J. (2004). A parsimonious arbitrage-free implied volatility parameterization with application to the valuation of volatility derivatives. In Global derivatives and risk management, Madrid.

Gneiting, T., \& Raftery, A. (2007). Strictly proper scoring rules, prediction, and estimation. Journal of the American Statistical Association, 102, 359-378.

Golubev, Y., Haerdle, W. K., \& Timofeev, R. (2014). Testing monotonicity of pricing kernels. Advances in Statistical Analysis, 98, 305-326.

Heston, S. L. (1993). A closed-form solution for options with stochastic volatility with applications to bond and currency options. Review of Financial Studies, 6, 327-343.

Jackwerth, J., \& Menner, M. (2017). Does the Ross recovery theorem work empirically? In Working paper, University of Konstanz.

Jackwerth, J. C. (2000). Recovering risk aversion from option prices and realized returns. Review of Financial Studies, 13, 433-451.

Jackwerth, J. C. (2004). Option-implied risk-neutral distributions and risk-aversion. Charlotteville, USA: Research Foundation of AIMR.

Jensen, C. S., Lando, D., \& Pedersen, L. H. (2017). Generalized recovery. Journal of Financial Economics, forthcoming.

Knüppel, M. (2015). Evaluating the Calibration of multi-step-ahead density forecasts using raw moments. Journal of Business \& Economic Statistics, 33, 270-281.

Linn, M., Shive, S., \& Shumway, T. (2017). Pricing kernel monotonicity and conditional information. Review of Financial Studies, forthcoming.

Mitchell, J., \& Hall, G. S. (2005). Evaluating, comparing and combining density forecasts using the KLIC with an application to the Bank of England and NIESR fan charts of inflation. Oxford Bulletin of Economics and Statistics, 67, 995-1033.

Polkovnichenko, V., \& Zhao, F. (2013). Probability weighting functions implied in options prices. Journal of Financial Economics, 107, 580-609.

Rosenberg, J. V., \& Engle, R. F. (2002). Empirical pricing kernels. Journal of Financial Economics, 64, $341-372$.

Ross, S. (2015). The recovery theorem. Journal of Finance, 70, 615-648.

Shackleton, M. B., Taylor, S. J., \& Yu, P. (2010). A multi-horizon comparison of density forecasts for the S\&P 500 using index returns and option prices. Journal of Banking and Finance, 34, 2678-2693. 